\title{
PROJECTABLE ALMOST COMPLEX CONTACT STRUCTURES
}

\author{
By D. E. BLAIR, S. IshiHARA AND G.D. LUdDEN
}

A complex manifold of complex dimension $2 m+1$ is said to be a complex contact manifold if it admits an open covering $\left\{u_{\alpha}\right\}$ such that on each $u_{\alpha}$ there is a holomorphic 1 -form $\omega_{\alpha}$ with $\omega_{\alpha} \wedge\left(d \omega_{\alpha}\right)^{m} \neq 0$ on $u_{\alpha} \cap u_{\beta} \neq \emptyset, \omega_{\beta}=f \omega_{\alpha}$ for some non-vanishing holomorphic function $f$. In general such a structure is not given by a global 1-form $\omega$; in fact this is the case for a compact complex manifold if and only if its first Chern class vanishes [6]. However, a complex contact manifold is the base space of a principal fibre bundle with 1-dimensional fibres and real contact structure. Homogeneous complex contact manifolds were studied by Boothby in [3].

It is also shown in [6] that the structural group of the tangent bundle of a Hermitian contact manifold $M$ is reducible to $(S p(m) \cdot S p(1)) \times U(1)$ where $S p(m)$ - $S p(1)=S p(m) \times S p(1) /\{ \pm I\}$ and hence equivalently $M$ admits the following local structure tensors. Let $F$ denote the almost complex structure and $g$ the Hermitian metric on $M$. Then each coordinate neighborhood admits tensor fields $G, H$ of type $(1,1)$ and vector fields $U, V$ with covariant forms $u$ and $v$ such that $(G, U, V, g)$ and $(H, U, V, g)$ are metric $f$-structures with complemented frames (see e.g. [1]), $F U=V$ and $G H=-H G=F+v \otimes U-u \otimes V$. In the overlap of coordinate neighborhoods we have

$$
\begin{array}{ll}
G^{\prime}=a G+b H, & u^{\prime}=a u+b v, \\
H^{\prime}=-b G+a H, & v^{\prime}=-b u+a v
\end{array}
$$

with $a^{2}+b^{2}=1$. Such a structure is called an almost complex contact structure [5] and our first project here will be to given an equivalent definition in terms of global tensor fields.

A standard example of a complex contact manifold is the odd-dimensional complex projective space $P C^{2 m+1}$. It is also well known that $P C^{2 m+1}$ is a fibre space over the quaternionic projective space $P \boldsymbol{H}^{m}$ with fibres $S^{2} \approx P \boldsymbol{C}^{1}$. In sections 3 and 4 we generalize this situation to a projectable almost complex contact structure on a Kählerian manifold.

\section{§ 1. Almost Complex Contact Structures}

In terms of the above local tensor fields $G, H, U, V$ we can define global Received June 14, 1976 
tensor fields $\Sigma$ of type $(1,3)$ and $S$ of type $(1,1)$. For local vector fields $X, Y, Z$ set

and

$$
\Sigma_{X Y} Z=g(G X, Y) G Z+g(H X, Y) H Z
$$

$$
S X=u(X) U+v(X) V \text {. }
$$

It is then easy to check using equations (0.1) that $\Sigma$ and $S$ are globally defined. Note also that $S$ is a projection tensor field of rank 2, i. e. $S^{2}=S$. For a unit vector $A \in T_{p} M$ with $S A=0$, let

$$
\sigma_{A}=\left\{B \in T_{p} M \mid g(A, B)=0,\|B\|=1, \underline{\Sigma}(A, B, A, B)=1\right\},
$$

where $\Sigma(X, Y, Z, W)=g\left(\Sigma_{X Y} Z, W\right)$ and $\left[\sigma_{A}\right]$ the subspace of $T_{p} M$ generated by $\sigma_{A}$.

The following properties of $\Sigma$ and $S$ are now easily deduced. 1)-8) are straightforward computations using equations (1.1) and (1.2) and elementary properties of metric $f$-structures. For 9$)$ given $A$ set $B=G A$ and it is easy to see that $B \in \sigma_{A}$.
1) $S F=F S$
2) $\Sigma_{X Y}=-\Sigma_{Y X}$
3) $\Sigma_{X Y}^{2}=\underline{\Sigma}(X, Y, X, Y)(-I+S)$
4) $\Sigma_{X Y} S=S \Sigma_{X Y}=0$
5) $\Sigma_{X Y F}=-F \Sigma_{X Y}$
6) $\Sigma_{X F Y} F=\Sigma_{X Y}$
7) $\underline{\Sigma}(X, Y, Z, W)=\underline{\Sigma}(Z, W, X, Y)$
8) $\Sigma_{X \Sigma_{Y Z} X} W=g(X,(I-S) X) \Sigma_{Y Z} W$
9) $\sigma_{A} \neq \emptyset$ for any unit vector $A$ with $S A=0$ and at any point $p$ of $M$.

Conversely we will show that an almost Hermitian manifold $M$ with structure tensors $(F, g)$ admitting global tensor fields $\Sigma$ and $S$ satisfying 1)-9) is an almost complex contact manifold. We first give several lemmas.

Lemma 1.1. For $B \in \sigma_{A}, \Sigma_{A B} A=B, S B=0$ and $\sigma_{A}$ is invariant under $F$.

Proof. Since $\underline{\Sigma}(A, B, A, B)=g\left(\Sigma_{A B} A, B\right)=1$ to show that $\Sigma_{A B} A=B$ it suffices to show that $\Sigma_{A B} A$ is a unit vector.

$$
g\left(\Sigma_{A B} A, \Sigma_{A B} A\right)=-g\left(\Sigma_{A B}^{2} A, A\right)=-g(-A+S A, A)=1
$$

by 2), 7) and 3), since $A$ is a unit vector and $S A=0$. Now $S B=S \Sigma_{A B} A=0$ by 4). Finally for the invariance by $F$,

$$
\Sigma_{A F B} A=-\Sigma_{A F B} F^{2} A=F \Sigma_{A B} A=F B,
$$

from which $\underline{\Sigma}(A, F B, A, F B)=1$ and $g(F B, A)=g\left(\Sigma_{A F B} A, A\right)=0$.

LEMMA 1.2. For any unt vector $B \in \sigma_{A}$ set $C=F B \in \sigma_{A}$, then 


$$
\Sigma_{A B} \Sigma_{A C}=F-S F .
$$

Proof. If we take an arbitrary vector $D \in T_{p} M$ then, using (3) and (6), we have

$$
\begin{aligned}
\Sigma_{A B} \Sigma_{A C} D & =\Sigma_{A B} \Sigma_{A F B} D=-\Sigma_{A B} \Sigma_{A F B} F^{2} D \\
& =-\Sigma_{A B} \Sigma_{A B} F D=(I-S) F D .
\end{aligned}
$$

LEMMA 1.3. For any orthonormal pair $\{B, C\} \in \sigma_{A}$,

$$
\Sigma_{A B} \Sigma_{A C}=-\Sigma_{A C} \Sigma_{A B} \text {. }
$$

Proof. First, using (3), we have

$$
\begin{aligned}
\Sigma_{A B+C}^{2} & =\Sigma(A, B+C, A, B+C)(-I+S) \\
& =2(-I+S) .
\end{aligned}
$$

On the other hand, we obtain

$$
\begin{aligned}
\Sigma_{A B+C}^{2} & =\left(\Sigma_{A B}+\Sigma_{A C}\right)^{2} \\
& =2(-I+S)+\left(\Sigma_{A B} \Sigma_{A C}+\Sigma_{A C} \Sigma_{A B}\right) .
\end{aligned}
$$

Thus we have $\Sigma_{A B} \Sigma_{A C}+\Sigma_{A C} \Sigma_{A B}=0$.

LEMMA 1.4. $\operatorname{dim}\left[\sigma_{A}\right]=2$.

Proof. Take $B$ and $C$ as in Lemma 1.1 and assume that there is a unit vector $D \in\left[\sigma_{A}\right]$ such that $D$ is orthogonal to $B$ and $C$. They by Lemmas 1.2 and 1.3 we have

and so

$$
\Sigma_{A B} \Sigma_{A C} \Sigma_{A D}=\Sigma_{A D} \Sigma_{A B} \Sigma_{A C}
$$

$$
(F-S F) \Sigma_{A D}=\Sigma_{A D}(F-S F) \text {. }
$$

Thus, using (1) and (4), we obtain

$$
F \Sigma_{A D}=\Sigma_{A D} F
$$

which contradicts (5). Therefore, $\left[\sigma_{A}\right]$ is necessarily of dimension 2 .

Lemma 1.5. For any vectors $B, C \in T_{p} M$, satısfying $\underline{\Sigma}(B, C, B, C)=1$, $\Sigma_{B C} A \in \sigma_{A}$.

Proof. Using (8), we have

$$
\Sigma_{A \Sigma_{B C} A} A=\Sigma_{B C} A,
$$

from which it follows that $\Sigma_{B C} A \in \sigma_{A}$. 
LEMmA 1.6. Take a unit vector $A \in T_{p} M$ with $S A=0$ and a unt vector $B \in \sigma_{A}$. Put $C=F B \in \sigma_{A}$. The $\Sigma_{A B} D$ and $\Sigma_{A C} D$ are orthonormal, where $D$ is an arbitrary unit vector at $p$ such that $S D=0$.

Proof. $g\left(\Sigma_{A B} D, \Sigma_{A B} D\right)=-g\left(\Sigma^{2}{ }_{A B} D, D\right)=g(D-S D, D)=1$ and similarly $\Sigma_{A C} D$ is also a unit vector. Finally

$$
\begin{aligned}
g\left(\Sigma_{A B} D, \Sigma_{A C} D\right) & =-g\left(\Sigma_{A B} D, \Sigma_{A F B} F^{2} D\right)=-g\left(\Sigma_{A B} D, \Sigma_{A B} F D\right) \\
& =g\left(\Sigma_{A B} D, F \Sigma_{A B} D\right)=0 .
\end{aligned}
$$

Summing up Lemmas 1.4, 1.5 and 1.6, we have

PROPOSITION 1. Take a unit vector $A \in T_{p} M$ such that $S A=0$ and a unit vector $B \in \sigma_{A}$. Put $C=F B \in \sigma_{A}$. Then, for any unit vector $D \in T_{p} M$ with $S D=0$, $\Sigma_{A B} D$ and $\Sigma_{A C} D$ form an orthonormal basis of $\left[\sigma_{D}\right]$.

Lemma 1.7. Take $A, B$ and $C$ as in Proposition 1. Then, for any $D, E$ $\in T_{p} M$,

$$
\Sigma_{D E}=\underline{\Sigma}(A, B, D, E) \Sigma_{A B}+\underline{\Sigma}(A, C, D, E) \Sigma_{A C} .
$$

Proof. When $D$ (or $E$ ) satisfies $S D=D$ (or $S E=E$ ), then both sides of the equation above vanish because of (4). So, $D$ and $E$ may be assumed to satisfy $S D=S E=0$ and also that $D$ and $E$ are unit. First, we consider the case in which $E$ is orthogonal to $\sigma_{D}$. Linearizing (3) we have $\Sigma_{X Y} \Sigma_{X Z}+\Sigma_{X Z} \Sigma_{X Y}$ $=2 \underline{\Sigma}(X, Y, X, Z)(-I+S)$. Thus if $Y \in \sigma_{D}, \Sigma_{D E}$ anti-commutes with $\Sigma_{D Y}$ and $\Sigma_{D F Y}$ and hence $\Sigma_{D E}$ commutes with $\Sigma_{D Y} \Sigma_{D F X}$ which by Lemma 1.2 is equal to $F-S F$. Therefore using (1) and (4)

$$
F \Sigma_{D E}=(F-S F) \Sigma_{D E}=\Sigma_{D E}(F-S F)=\Sigma_{D E} F
$$

from which by (5) and the non-singularity of $F$ we have $\Sigma_{D E}=0$ and again both sides of the above equation vanish.

Finally we consider the case where $E \in \sigma_{D}$. For simplicity set $a=g\left(\Sigma_{A B} D, E\right)$ and $b=g\left(\Sigma_{A C} D, E\right)$. Then as $\left\{\Sigma_{A B} D, \Sigma_{A C} D\right\}$ is an orthonormal basis of $\left[\sigma_{D}\right]$,

Using (8) we have

$$
E=a \Sigma_{A B} D+b \Sigma_{A C} D \text {. }
$$

Using (8) again

$$
\begin{aligned}
\Sigma_{D E} A & =a \Sigma_{D \Sigma_{A B} D} A+b \Sigma_{D \Sigma_{A C} D} A \\
& =a \Sigma_{A B} A+b \Sigma_{A C} A \\
& =a B+b C .
\end{aligned}
$$

$$
\Sigma_{D E}=\Sigma_{A \Sigma_{D E} A}=a \Sigma_{A B}+b \bar{\Sigma}_{A C},
$$

which is the desired formula. 
Take a suitable coordinate neighborhood $u$ of an arbitrary point $p$ of $M$ and a unit vector field $A$ in $u$. Then there is in $u$ a unit vector field $B$ belonging to $\sigma_{A}$ at each point of $u$. On putting $C=F B \in \sigma_{A}$ we define locally in $u$ two tensor fields $G$ and $H$ of type $(1,1)$ respectively by

$$
G=\Sigma_{A B}, \quad H=\Sigma_{A C} .
$$

Then setting $F^{H}=F-F S$ and using (3) and (4) and Lemma 1.3, we have

$$
\begin{aligned}
& \left(F^{H}\right)^{2}=G^{2}=H^{2}=-I+S, \\
& G H=-H G=F^{H}, H F^{H}=-F^{H} H=G, F^{H} G=-G F^{H}=H, \\
& F^{H} S=S F^{H}=G S=S G=H S=S H=0 .
\end{aligned}
$$

Next, (1), (2), and (7) imply

$$
\begin{aligned}
& g\left(F^{H} X, Y\right)=-g\left(F^{H} Y, X\right), \\
& g(G X, Y)=-g(G Y, X), g(H X, Y)=-g(H Y, X),
\end{aligned}
$$

for all $X$ and $Y$. By Lemma 1.7, a local expression for $\Sigma_{X Y}$ in $u$ is the following

$$
\Sigma_{X Y}=g(G X, Y) G+g(H X, Y) H .
$$

We now take another coordinate neighborhood $u^{\prime}\left(u \cap u^{\prime} \neq \emptyset\right)$ and define $G^{\prime}$ and $H^{\prime}$ as in $u$, say $G^{\prime}=\Sigma_{A^{\prime} B^{\prime}}$ and $H^{\prime}=\Sigma_{A^{\prime} C^{\prime}}$. By the formula of Lemma 1.7

$$
\begin{aligned}
& \Sigma_{A^{\prime} B^{\prime}}=\underline{\Sigma}\left(A, B, A^{\prime}, B^{\prime}\right) \Sigma_{A B}+\underline{\Sigma}\left(A, C, A^{\prime}, C^{\prime}\right) \Sigma_{A C} . \\
& \Sigma_{A^{\prime} C^{\prime}}=\underline{\Sigma}\left(A, B, A^{\prime}, C^{\prime}\right) \Sigma_{A B}+\underline{\Sigma}\left(A, C, A^{\prime}, C^{\prime}\right) \Sigma_{A C} .
\end{aligned}
$$

Setting $a=\underline{\Sigma}\left(A, B, A^{\prime}, B^{\prime}\right)$ and $b=\underline{\Sigma}\left(A, C, A^{\prime}, B^{\prime}\right)$ we have that

and

$$
1=g\left(\Sigma_{A^{\prime} B^{\prime}} A^{\prime}, B^{\prime}\right)=\underline{\Sigma}\left(A, B, A^{\prime}, B^{\prime}\right)^{2}+\underline{\Sigma}\left(A, C, A^{\prime}, B^{\prime}\right)^{2}=a^{2}+b^{2}
$$

$$
\begin{aligned}
\underline{\Sigma}\left(A, C, A^{\prime}, C^{\prime}\right) & =-g\left(\Sigma_{A F B} F^{2} A^{\prime}, F B^{\prime}\right)=-g\left(\Sigma_{A B} F A^{\prime}, F B^{\prime}\right) \\
& =g\left(F \Sigma_{A B} A^{\prime}, F B^{\prime}\right)=g\left(\Sigma_{A B} A^{\prime}, B^{\prime}\right)=a, \\
\underline{\Sigma}\left(A, B, A^{\prime}, C^{\prime}\right) & =-g\left(\Sigma_{A F B} F A^{\prime}, F^{2} C^{\prime}\right)=-g\left(F \Sigma_{A C} A^{\prime}, F B^{\prime}\right) \\
& =-g\left(\Sigma_{A C} A^{\prime}, B^{\prime}\right)=-b,
\end{aligned}
$$

so that $G^{\prime}=a G+b H$ and $H^{\prime}=-b G+a H$.

Theorem 1. Let $(M, G, F)$ be an almost Hermitian manfold. Then $M$ is an almost complex contact manifold if and only if $M$ admits a global tensor field $\Sigma$ of type (1,3) and a projection tensor field $S$ of rank 2 satısfying 1)-9). 


\section{§ 2. Horizontal and Vertical Tensors}

Given a vector field $X$ on an almost Hermitian manifold $(M, g, F)$ with almost complex contact structure $(g, F, \Sigma, S), X^{V}=S X$ and $X^{H}=X-X^{V}$ will be called the vertical and the horizontal parts of $X$, respectively. For a 1-form $\omega$, $\omega^{V}=\omega^{\circ} S$ and $\omega^{H}=\omega-\omega^{V}$ will be called the vertical and the horizontal parts of $\omega$, respectively. We now define, for a function $f, f^{H}=f^{V}=f$. Then we easily have

$$
\begin{aligned}
& (f X+h Y)^{H}=f^{H} X^{H}+h^{H} Y^{H},(f X+h Y)^{V}=f^{V} X^{V}+h^{V} Y^{V}, \\
& (f \omega+h \pi)^{H}=f^{H} \omega^{H}+h^{H} \pi^{H},(f \omega+h \pi)^{V}=f^{V} \omega^{V}+h^{V} \pi^{V},
\end{aligned}
$$

where $f, h$ are arbitrary functions and $\omega, \pi$ are arbitrary 1 -forms.

We now define the horizontal part $T^{H}$ of an arbitrary tensor field $T$. Assume that the operation of taking the horizontal part satisfies

$$
(P+Q)^{I I}=P^{H}+Q^{H},(P \otimes U)^{H}=P^{H} \otimes U^{H},
$$

where $P$ and $Q$ are arbitrary tensor fields of the same type and $U$ another arbitrary tensor field, then by using (2.1) we can inductively define the horizontal part $T^{H}$ of an arbitrary tensor field $T$ on $M$.

\section{§3. Almost Complex Contact Structures which are Projectable}

The Riemannian connection is denoted by $\nabla$ in a Kählerian manifold $M$ with almost complex contact structure $(g, F, \Sigma, S)$. We define a tensor field $P$ of type: $(1,2)$ by

$$
P_{X} Y=\left(\left(\nabla_{Y} S\right) X\right)^{H}
$$

Note that

$$
S P_{X}=0 \text {. }
$$

Next, differentiating covariantly $S^{2}=S$ we have

$$
P_{S X}=P_{X}
$$

and differentiating covariantly (1)

$$
P_{F X}=F P_{X} .
$$

Lemma 3.1. When $P=0$, a Kählerian manfold $M$ of complex dimension $2 m+1$ with almost complex contact structure $(g, F, \Sigma, S)$ is locally a product of two Kählerian manifolds of complex dimensions $2 m$ and 1 respectively.

Proof. If $P=0,(3.1)$ implies

$$
\left(\nabla_{Y}(S X)\right)^{H}=\left(\left(\nabla_{Y} S\right) X\right)^{H}=0,
$$


which means that the distribution determined by $S$ and its complement are parallel. This with $S F=F S$ proves the lemma.

We now consider the following conditions :

(P1) for any vector $A \in T_{p} M$, there are two vectors $B, C \in T_{p} M$ such that $P_{A}=\Sigma_{B C}, \underline{\Sigma}(B, C, B, C)=a g(S A, S A)$ with constant $a$;

(P2) $\quad\left(\nabla_{S X} \underline{\Sigma}\right)^{H}=0$.

When an almost complex contact structure $(g, F, \Sigma, S)$ satisfies the conditions (P1) and (P2), it is said to be projectable.

In this section, the almost complex contact structure $(g, F, \Sigma, S)$ is assumed to be projectable. Then 3$)-4$ ) and (P1) imply

$$
P_{X}{ }^{2}=\operatorname{ag}(S X, S X)(-I+S)
$$

for some $a$ and

$$
P_{X} S=0
$$

is equivalent to

$$
S\left(\nabla_{S Y} S\right)=\nabla_{S Y} S
$$

Thus we now have from (1) and (3.7)

Proposition 2. In a Kählerian manifold $M$ with almost complex contact structure $(g, F, \Sigma, S)$ which satisfies (P1), the distribution determined by $S$ is integrable and each of its integral submanifolds is totally geodesic and holomorphic.

Since (P1) is satisfied, restricting ourselves to a coordinate neighborhood $u$ in which (1.4) is established, we find

$$
P_{X}=c(u(X) G+v(X) H)
$$

with local 1 -forms $u$ and $v$ defined in $u$, where the associated vector fields $U$ of $u$ and $V$ of $v$ satisfy $\|U\|^{2}=\|V\|^{2}=1, g(U, V)=0$, i. e.,

$$
S=u \otimes U+v \otimes V \text {. }
$$

(3.8) implies that

$$
(\nabla(S X))^{H}=c(u(x) G+v(x) H) .
$$

The fundamental 2-form $\Phi$ of the Kählerian manifold $(M, g, F)$ is defined by $\Phi(X, Y)=g(F X, Y)$. We now define in $M$ a tensor field $\underline{\Lambda}$ of type $(0,4)$ by

$$
\underline{\Lambda}=\Phi^{H} \otimes \Phi^{H}+\underline{\Sigma},
$$

which is horizontal, that is, $\underline{\Lambda}^{H}=\underline{\Lambda}$. Then, using (1.3) and (3.8), we can verify that in $u$ 


$$
P_{U} \cdot \underline{\Lambda}=0, \quad P_{V} \cdot \underline{\Lambda}=0,
$$

where $P_{X}$. denotes the action of $P_{X}$ as a derivation. Thus, using (3.9), we obtain

$$
P_{X} \cdot \underline{1}=0
$$

Since $\nabla F=0$, we find

$$
\left(\nabla_{S X} \underline{\Lambda}\right)^{H}=0
$$

as a consequence of (P2). As is well known, the Lie derivative $\mathcal{L}_{S X} \underline{\Lambda}$ is given by

$$
\mathcal{L}_{S_{X}} \underline{\Lambda}=\nabla_{S_{X}} \underline{\Lambda}+P_{X} \cdot \underline{\Lambda}
$$

(See e.g. Yano [8]). Thus we have

$$
\left(\mathcal{L}_{X} V \underline{\Lambda}\right)^{H}=0 .
$$

LEMMA 3.2. If an almost complex contact structure $(g, F, \Sigma, S)$ is proiectable, then

$$
\left(\mathcal{L}_{X} V \underline{\Lambda}^{I I}\right)^{H}=0
$$

On the other hand, by Proposition 2, each integral submanifold of the distribution determined by $S$ is totally geodesic. Thus we have (see Ishihara and Konishi [5]) then

LEMMA 3.3. If an almost complex contact structure $(g, F, \Sigma, S)$ is projectable,

We now put

$$
\left(\mathcal{L}_{X} V g^{H}\right)^{I I}=0 \text {. }
$$

$$
\Lambda=\Phi \otimes F+\Sigma .
$$

Then Lemmas 3.2 and 3.3 imply then

Lemma 3.4. If an almost complex contact structure $(g, F, \Sigma, S)$ is projectable,

$$
\left(\mathcal{L}_{X} \nabla \Lambda^{H}\right)^{H}=0 .
$$

\section{$\S 4$. Submersion of a Kählerian Manifold with Almost Complex Contact Structure}

Let $(M, G, F)$ be a Kählerian manifold of complex dimension $2 m+1$ with almost complex contact structure $(g, F, \Sigma, S)$, which is projectable, and $\tilde{M}$ a manifold of real dimension $4 \mathrm{~m}$. Suppose that there is a differential mapping $\pi: M \rightarrow \tilde{M}$ which is of rank $4 m$ everywhere and satisfies $\pi(M)=\tilde{M}$ and that for each point $p$ of $\tilde{M}, \pi^{-1}(p)$ is a connected integral submanifold of the distribution 
determined by $S$. In such a case, the Kählerian manifold $M$ with almost complex contact structure is said to have a fibred Riemannian structure $\pi: M \rightarrow \tilde{M}$ and $\tilde{M}$ is called the base space. When $M$ is compact and the distribution $\mathscr{D}$ determined by $S$ is regular, $M$ has a fibred Riemannian structure if $\tilde{M}$ is defined as the set of all maximal integral submanifolds of $\mathscr{D}, \pi: M \rightarrow \tilde{M}$ being defined by $\pi(p)=\mathscr{D} p$, $p \in M$, where $\mathscr{D} p$ is the maximal integral submanifold passing through $p$, and $\tilde{M}$ is naturally topologized.

Consider a Kählerian manifold $M$ with almost complex contact structure $(g, F, \Sigma, S)$, which is projectable, and with fibred Riemannian structure $\pi: M \rightarrow \tilde{M}$. Then, taking account of arguments developed in [5], we see by Lemma 3.4 that the tensor field $\Lambda$ is projectable in $M$ and its projection is a tensor field $\tilde{\Lambda}$ of type $(1,3)$ in the base space $\tilde{M}$. The metric tensor $g$ in $M$ is, by Lemma 3.3, projectable and its projection $\tilde{g}$ defines a Riemannian structure on $\tilde{M}$. Thus, (2)-(9) implies that $(\tilde{g}, \tilde{\Lambda})$ is an almost quaternionic structure in the base space $\tilde{M}$ (see Blair and Showers [2]). Thus, summing up, we have

THEOREM 2. Suppose that $(M, g, F)$ is a Kählernan manifold with almost complex contact structure $(g, F, \Sigma, S)$, which is projectable. Assume moreover that $(M, g, F)$ has a fibred Riemannian structure $\pi: M \rightarrow \tilde{M}$. Then $(\tilde{g}, \tilde{\Lambda})$ is an almost quaternionc structure in the base space $\tilde{M}$, where $\tilde{g}$ and $\tilde{\Lambda}$ are the projections of $g$ and $\Lambda$, respectuvely.

If in a Kählerian manifold $M$ satisfying the conditions given in Theorem 2

$$
\left(\nabla \Lambda^{H}\right)^{H}=0
$$

holds, then the projection $\tilde{\Lambda}$ of $\Lambda$ in $\tilde{M}$ is covariantly constant. Thus in such a case $(\tilde{g}, \tilde{\Lambda})$ is a quaternionic Kählerian structure (see Ishihara [4]). Thus we have

THEOREM 3. If, an a Kählerian manifold $M$ satisfynng the conditions given in Theorem 2, $\left(\nabla \Lambda^{H}\right)^{H}=0$, then $(\tilde{g}, \tilde{\Lambda})$ is a quaternionic Kählerian structure in the base space $\tilde{M}$.

Taking account of Lemma 3.1, we easily have

Proposition 3. If a Kählerian manifold $M$ of complex dimension $2 m+1$ with almost complex contact structure $(g, F, \Sigma, S)$, which is projectable, satısfies the condition $P=0$, then $M$ is locally a product of Kählerian manfolds $\left(M_{1}, g_{1}, F_{1}\right)$ of complex dimension $2 m$ and $\left(M_{2}, g_{2}, F_{2}\right)$ of complex dimension 1 , where $M_{1}$ admits quaternon structure $\left(g_{1}, \Lambda_{1}\right)$.

Proposition 4. If, in a Kählerian manfold $M$ satısfying the conditions given in Proposition $3\left(\nabla \Lambda^{H}\right)^{H}=0$ then $M_{1}$ admits a quatermonic Kählerian structure $\left(g_{1}, \Lambda_{1}\right)$ wrth vanushng Ruccu tensor (see Ishihara [4]). 


\title{
BIBLIOGRAPHY
}

[1] D. E. BLAir, Geometry of Manifolds with Structural Group $u(n) \times O(S)$, J. Diff. Geom. 4 (1970) 155-167.

[2] D. E. Blair and D. K. Showers, A Note on Quaternionic Geometry, to appear.

[3] W. M. Bоотнвy, Homogeneous Complex Contact Manifolds, Proc. Symposia in Pure Math. of A. M. S., Vol. III, Diff. Geom., 1961, 144-154.

[4] S. Ishihara, Quaternion Kählerıan manifolds, J. Diff. Geom. 9 (1974) 483-500.

[5] S. Ishihara AND M. Konishi, Complex Almost Contact Structures and Fiberings, to appear.

[6] S. Kobayashi, Remarks On Complex Contact Manifolds, Proc. of A. M.S. 10 (1959), 164-167.

[7] S. SASAKI, Almost Contact Manifolds I, II, III, Lecture Notes, Tôhoku University, 1965.

[8] K. YANo, Theory of Lie Derivatives, North Holland, Amsterdam, 1957.

\author{
Michigan State University \\ EAST LANSING \\ Department of Mathematics \\ Tokyo Institute of Technology \\ Michigan State University \\ EAST LANSING
}

